\title{
Publisher Correction: The condensin holocomplex cycles dynamically between open and collapsed states
}

Je-Kyung Ryu, Allard J. Katan, Eli O. van der Sluis, Thomas Wisse, Ralph de Groot, Christian H. Haering and Cees Dekker (D) Correction to: Nature Structural \& Molecular Biology https://doi.org/10.1038/s41594-020-0508-3, published online 28 September 2020. In the version of this article initially published online, the title of the Fig. 4 caption, "DNA loops extruded by condensing," was incorrect. The correct title is "DNA loops extruded by condensin." The error has been corrected in the print, PDF and HTML versions of the article.

Published online: 8 October 2020

https://doi.org/10.1038/s41594-020-00524-y

( ) The Author(s), under exclusive licence to Springer Nature America, Inc. 2020 